\title{
What maketh the man?
}

Many behavioural and physiological differences between the sexes result from the effects of steroids during development. In the brain of males, but not females, oestradiol is formed from testosterone and has a role in establishing male-specific neural circuitry. A full understanding of the mechanisms that underlie oestradiol's effects on circuit formation was lacking; however, a study by Schwarz and colleagues now describes the signalling pathway that mediates its effects on dendritic spine formation in the hypothalamus.

Hypothalamic neurons of newborn male rats have more dendritic spines, and presumably make more synaptic connections, than those of females. However, if newborn females are treated with oestradiol, their spine number increases until it is equivalent to that of males within hours. Here, the authors set out to investigate the mechanisms that underlie oestradiol's effects on spine formation.

The authors demonstrated that the effects of oestradiol on spine formation in females could be replicated by treatment with NMDA ( $N$-methyl-D-aspartate) and blocked by glutamate receptor antagonists, implicating glutamate receptor activity in oestradiol's effects. However, oestradiol did not alter the number or function of glutamate receptors in the female hypothalamus, suggesting that its effects occur at the level of transmitter release.

The authors measured the pairedpulse ratio, which is inversely related to presynaptic glutamate release, in hypothalamic neurons in brain slices taken from treated and control females. They also treated cultured female hypothalamic neurons with oestradiol and measured the rate at which a membrane dye was released from presynaptic terminals in response to depolarization. Both measurements showed that oestradiol increases glutamate release within 2 hours of treatment. Binding of oestradiol to estrogen receptors (ERs) was crucial for this effect, as blocking ERs prevented the increased glutamate release.

ER activation is classically associated with the regulation of gene expression, but the rapid effect of oestradiol on glutamate release makes such a downstream mechanism unlikely in this case. ERs can also directly activate some protein kinases, and the authors showed that phosphatidylinositol 3-kinase (PI3K) activity was increased within 1 hour of oestradiol treatment in cultured hypothalamic neurons. Furthermore, blocking PI3K activity prevented the effects of oestradiol on both glutamate release and spine formation.

Next the authors considered the mechanisms by which an increase in glutamate release, and thus in postsynaptic glutamate receptor activation, alters spine formation. An increase in the activity of several mitogen-activated protein kinases (MAPKs) — including MEK1/2 and c-Jun kinase - occurred 3 hours after oestradiol treatment in cultured

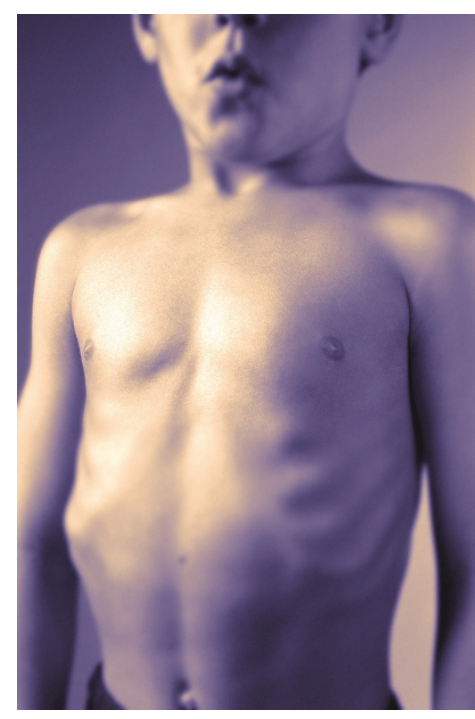

neurons. This effect relied on glutamate receptor activation. Blocking MAPK activity abolished the effects of oestradiol on spine formation in female rats.

From these results, the signalling pathway by which oestradiol regulates spine formation begins to emerge: the activation of ERs by oestradiol increases PI3K activity, which enhances presynaptic glutamate release. This in turn increases the activation of postsynaptic glutamate receptors, triggering spine formation through a MAPKdependent mechanism. Although some details of the pathway remain to be uncovered, the findings illustrate how trans-synaptic communication between cells can shape neuronal circuitry during development.

Katherine Whalley

\section{ORIGINAL RESEARCH PAPER}

Schwarz, J. M. et al. Estradiol induces hypothalamic dendritic spines by enhancing glutamate release: a mechanism for organizational sex differences. Neuron 58, 584-598 (2008) 